\title{
Anterior minimally invasive bridge-plate technique for treatment of humeral shaft nonunion
}

\author{
Paulo Roberto Vilaça Jr. • Marcelo Koh Uezumi
}

Received: 25 July 2011/ Accepted: 6 June 2012/Published online: 21 June 2012

(C) The Author(s) 2012. This article is published with open access at Springerlink.com

\begin{abstract}
Background The present study introduces a new surgical technique and the results of a case series of patients with humeral shaft nonunion.

Materials and methods Fifteen patients with diagnosis of diaphyseal nonunion of humerus were operated by a bridge-plate technique. A $4.5-\mathrm{mm}$ plate is slid on the anterior surface of the humerus, submuscular to the brachial muscle. With the plate over the anterior surface of the humerus, screws are inserted from anterior to posterior on the ends of the plate. When there is a small bone gap, an iliac autologous graft is inserted. Minimum follow-up was 1 year.

Results Bone healing was obtained in all patients: 1.5 months postoperatively in 11 patients, 2 months in 3 patients, and 3 months in 1 patient. There were no postoperative infections, there was one case with loosening of the screws and plate, and there were no nerve injuries. Conclusions The present technique avoids wide dissection, radial nerve isolation, and periosteum stripping. The anterior minimally invasive bridge-plate technique for treatment of humeral shaft nonunion is a safe procedure and obtained bone healing in all patients in this series.
\end{abstract}

Keywords Nonunion $\cdot$ Humerus $\cdot$ Bridge plate $\cdot$ MIPO

Ethical Standard: This study was approved by the Regional Ethics Committee (Comitê de Ética em Pesquisa da Secretaria Municipal de Saúde de São Paulo—protocol number CAAE: 254.0.162.000-10).

P. R. Vilaça Jr. (凹) • M. K. Uezumi

Brazilian Orthopaedics and Traumatology Society-SBOT,

Associação Beneficente Nossa Senhora do Pari,

Hospital Nossa Senhora do Pari, Rua Hannemann,

234, São Paulo, SP, Brazil

e-mail: prvjbr@ig.com.br; paulo.vilaca@usp.br

\section{Introduction}

Humeral nonunion is a condition resulting from lack of healing at the fracture site often associated with alteration of the local biological potential. Due to the preoperative conditions and the difficulties inherent to the surgery, treatment is a challenge.

Ring et al. [10] demonstrated that the most important factor to achieve bone healing in nonunion is use of a careful, biologically and mechanically adequate technique.

Using a surgical technique that respects the basic principles of less soft tissue dissection, preservation of blood supply, and immediate rehabilitation of the operated limb, the present study introduces a new surgical technique for treatment of humeral shaft nonunion and the results of a case series of patients.

\section{Materials and methods}

Fifteen patients ( 6 women and 9 men) with diagnosis of diaphyseal nonunion of humerus were operated on by the same surgeon between July 2008 and June 2010.

The Regional Ethics Committee approved this study (protocol number CAAE: 254.0.162.000-10), and all subjects signed informed consent. The study has been performed in accordance with the ethical standards laid down in the 1964 Declaration of Helsinki.

Mean patient age was $51.5 \pm 21.1$ years (range 23-85 years) with nonunion for an average of $11.5 \pm 5.4$ months (range 6-24 months). Seven patients had undergone previous surgery after the initial trauma. Of these, four had already been reoperated for the established nonunion, unsuccessfully receiving plates and screws with autologous bone grafting in this second operation (Table 1). 
Table 1 Preoperative data

\begin{tabular}{llllll}
\hline Patient & $\begin{array}{l}\text { Age } \\
\text { (years) }\end{array}$ & Type & $\begin{array}{l}\text { Previous } \\
\text { treatment }\end{array}$ & $\begin{array}{l}\text { Nonunion duration } \\
\text { (months) }\end{array}$ & Complaint \\
\hline 1 & $54 / \mathrm{M}$ & Hypertrophic & Conservative & 9 & Instability \\
2 & $62 / \mathrm{M}$ & Atrophic & Plate fixation & 12 & Instability \\
3 & $54 / \mathrm{M}$ & Atrophic & Plate fixation & 14 & Instability \\
4 & $35 / \mathrm{M}$ & Atrophic & Nail & 6 & Pain \\
5 & $29 / \mathrm{M}$ & Atrophic & Plate fixation & 24 & Pain \\
6 & $68 / \mathrm{F}$ & Atrophic & Conservative & 6 & Pain + instability \\
7 & $26 / \mathrm{M}$ & Atrophic & Nail & 12 & Pain + instability \\
8 & $85 / \mathrm{F}$ & Atrophic & Conservative & 12 & Pain + instability \\
9 & $24 / \mathrm{F}$ & Hypertrophic & Nail & 6 & Pain \\
10 & $36 / \mathrm{M}$ & Hypertrophic & Conservative & 6 & Pain \\
11 & $71 / \mathrm{M}$ & Atrophic & Conservative & 12 & Pain + instability \\
12 & $73 / \mathrm{F}$ & Atrophic & Conservative & 12 & Instability \\
13 & $77 / \mathrm{F}$ & Atrophic & Plate fixation & 18 & Pain + instability \\
14 & $55 / \mathrm{M}$ & Atrophic & Conservative & 6 & Pain + instability \\
15 & 23/F & Atrophic & Plate fixation & 18 & Instability \\
\hline
\end{tabular}

The inclusion criterion was diaphyseal nonunion of humerus. Cases with active infection were excluded. Nonunion was defined as lack of bone healing at least 6 months after the fracture [3]. The type of nonunion was classified by radiographic standards [3] as being atrophic or hypertrophic. In this series, 12 cases were atrophic and 3 were hypertrophic.

Four patients presented with osteoporotic bones. In these patients, no good screw fixation could be achieved so a locking plate (LCP) and screws were used.

Clinical and radiographic evaluations were done $0.5,2$, 3 , 6, and 12 months postoperatively. After this period, follow-ups were done every 6 months. University of California, Los Angeles (UCLA) score system [2] was adopted for shoulder function clinical evaluation. The bone healing criteria were clinical absence of pain and mobility, and radiographic presence of healed cortices.

\section{Statistical analysis}

A related-sample Wilcoxon signed-rank test was used to compare pre- and postoperative UCLA scale values and pre- and postoperative elbow function. Data are presented as average \pm standard deviation (SD). Statistical significance was set at 0.05 .

Minimum follow-up was 1 year with a range of $1-3.5$ years.

\section{Operative technique}

The patient is operated in supine position, with the bone graft removed from the contralateral iliac crest in case of atrophic nonunion.

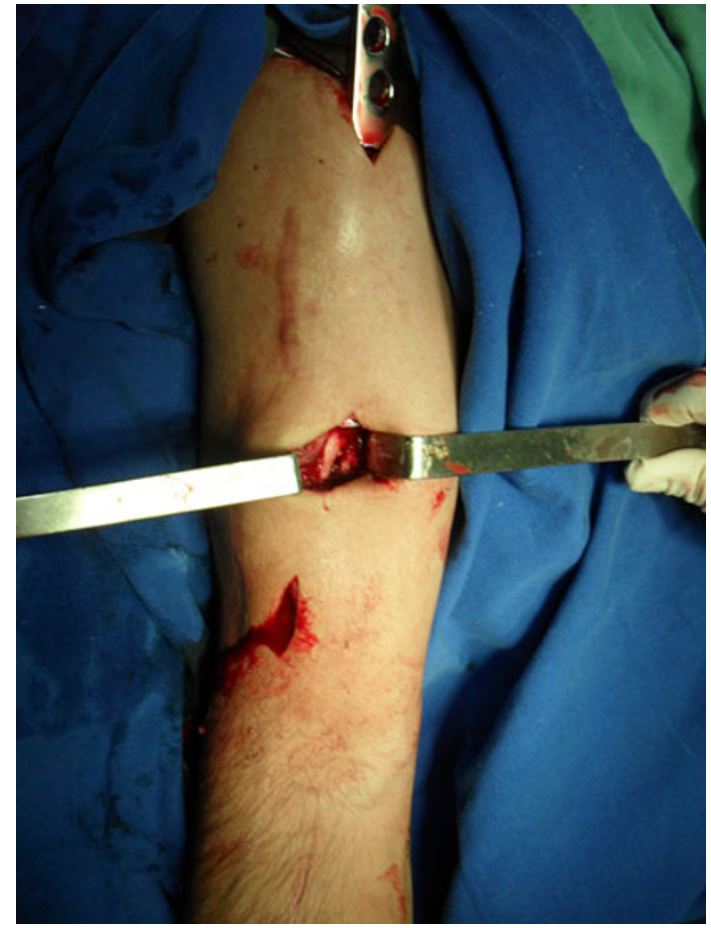

Fig. 1 With the plate slid in over the anterior surface of the humerus, the screws are inserted from anterior to posterior on the extremities of the plate. Through an auxiliary incision on the anterior surface of the humerus, the bone graft is inserted into the atrophic nonunion site. Distally the radial nerve is not seen and is protected laterally by muscles

In the distal humerus region, a 4-cm anterior longitudinal incision is made on the lateral border of the biceps. Between the biceps and the brachioradialis muscles, the brachial muscle can be accessed more deeply. A blunt longitudinal midline opening is made in the fibers of the brachial muscle. 


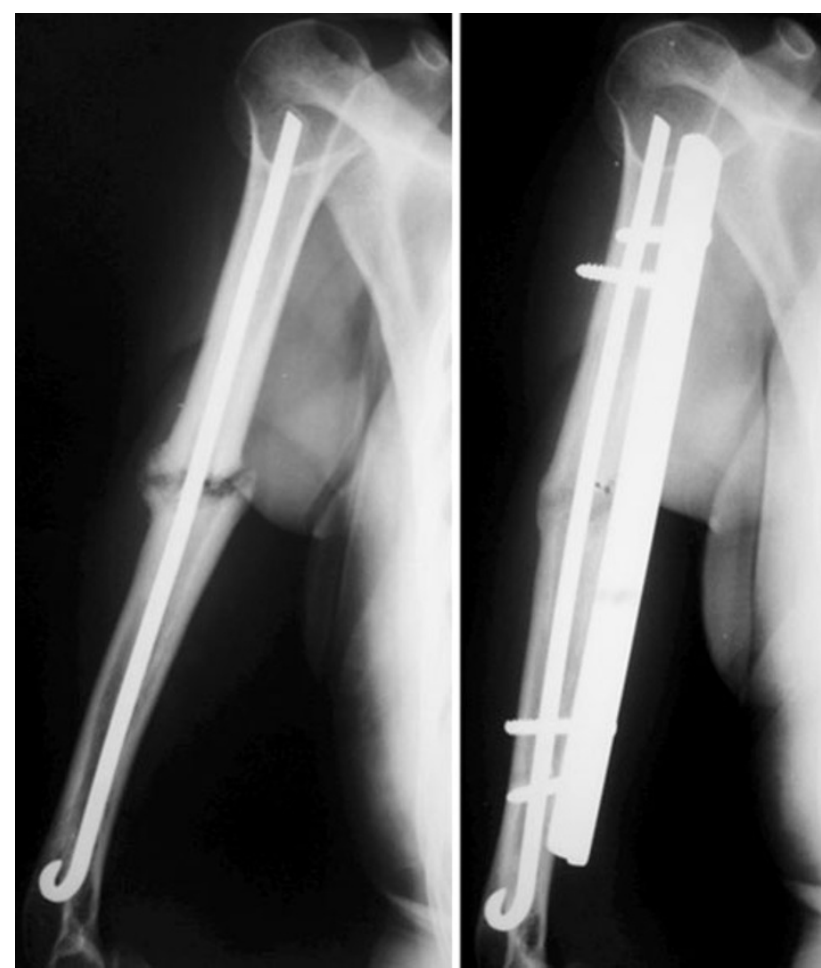

Fig. 2 Hypertrophic nonunion case. The plate is passed and fixed over the anterior surface of the humerus. The Rush nail was maintained because there was no complaint and it would be difficult to remove without bone aggression

The brachialis muscle is innervated at the lateral surface by the radial nerve and medially by the musculocutaneous nerve. This anatomical detail allows its longitudinal opening without any harm to nerves or loss of function; then, access to the anterior surface of the humerus is easily achieved. Through this opening, the screws will be fixed distally.

Distally, the muscles including the lateral part of the brachialis and the brachioradialis protect the radial nerve, which is neither seen nor dissected in this approach. Proximally, a 4-cm incision is made between the medial border of the deltoid and the lateral border of the biceps. The interval between these two muscles is the location to slide in and fix the plate. A 4.5-mm dynamic compression plate (DCP) (or LCP in osteoporotic cases) must be slid on the anterior surface of the humerus, submuscular to the brachial muscle [7] (Fig. 1). The direction in which the plate is slid differs according to each case. Distally, one must be careful not to violate the coronoid fossa.

With the plate slid over the anterior surface of the humerus, the screws (two or three in each fragment normally) are inserted from anterior to posterior on the ends of the plate. In case of hypertrophic nonunion, no further surgery is needed (Fig. 2).

In case of atrophic nonunion, a $3-\mathrm{cm}$ auxiliary incision is made over the nonunion site on the anterior surface of the humerus with direct dissection to the site. Fibrous tissue
Table 2 Postoperative data

\begin{tabular}{lllll}
\hline Patient & $\begin{array}{l}\text { Time to } \\
\text { union } \\
\text { (weeks) }\end{array}$ & $\begin{array}{l}\text { Shoulder } \\
\text { function } \\
\text { (UCLA)/ } \\
\text { Ellman } \\
\text { criteria }\end{array}$ & $\begin{array}{l}\text { Elbow } \\
\text { function } \\
\text { extension/ } \\
\text { flexion }\left(^{\circ}\right)\end{array}$ & $\begin{array}{l}\text { Final } \\
\text { complaint }\end{array}$ \\
\hline 1 & 6 & 35/excellent & $0 / 135$ & None \\
2 & 6 & 33/good & $10 / 122$ & None \\
3 & 8 & 25/satisfactory & $40 / 110$ & Iliac crest pain \\
4 & 12 & 27/satisfactory & $30 / 120$ & None \\
5 & 6 & 33/good & $22 / 130$ & Iliac crest pain \\
6 & 6 & 27/satisfactory & $38 / 114$ & Iliac crest pain \\
7 & 6 & 35/excellent & $0 / 110$ & None \\
8 & 6 & 22/satisfactory & $30 / 100$ & None \\
9 & 6 & 35/excellent & $5 / 130$ & None \\
10 & 6 & 27/satisfactory & $8 / 130$ & None \\
11 & 6 & 32/good & $0 / 130$ & Iliac crest pain \\
12 & 6 & 26/satisfactory & $16 / 135$ & Iliac crest pain \\
13 & 8 & 29/good & $40 / 110$ & None \\
14 & 8 & 19/poor & $18 / 128$ & Diffuse pain on \\
& & & & arm \\
15 & 6 & 35/excellent & $0 / 128$ & None \\
\hline
\end{tabular}

is removed, and the bone prepared. With a small bone gap (less than $1 \mathrm{~cm}$ ), a cancellous iliac autologous graft is inserted. With greater bone loss, the structured bone graft is "press-fitted" into the nonunion without the need for complementary fixation.

\section{Rehabilitation}

Immediately postoperatively, patients were directed to use the limb in their usual activities, and to use a sling only for pain control in the first 5 days if necessary, mainly at night when sleeping. Use of immobilization was not advised after this period.

All patients were recommended to move the elbow and shoulder, avoiding stiffness.

After bone healing, a rehabilitation program was performed. The first aim was to gain full mobility, then proprioception, and finally muscular strengthening. The total rehabilitation period depends on the progression of each patient. The final goal is to restore full range of motion and strength without pain.

\section{Results}

Bone healing was obtained in all patients: after 1.5 months postoperatively in 11 patients, 2 months in 3 patients, and 3 months in 1 patient (Table 2). There were no postoperative infections. There was one case with loosening of the screws and plate. There were no nerve injuries. 


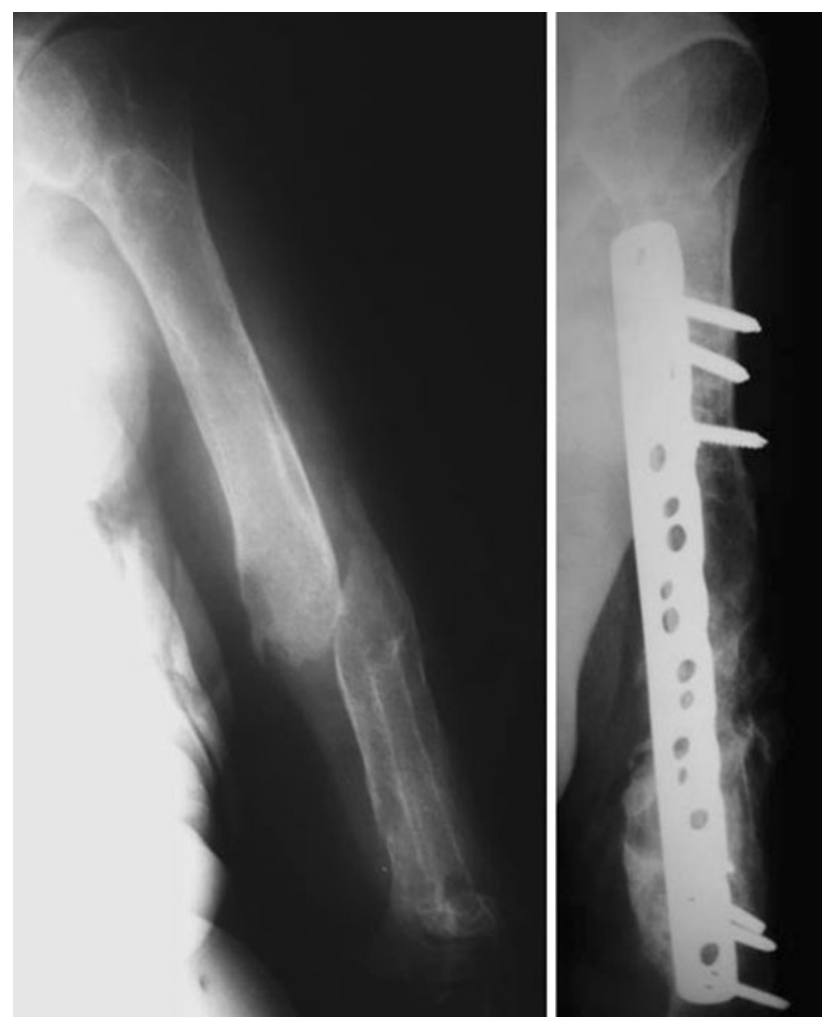

Fig. 3 Atrophic nonunion in an osteoporotic bone (left). After cleaning and grafting focus and bridge-plate fixation with locking plate and screws, the nonunion is healed (right)

The case with hardware loosening was reoperated using the same bridge-plate technique. Plate and screws were removed, better reduction was performed, and new bone graft applied. Bone healing was obtained in 2 months. As better reduction was achieved and there was good fixation of the screws, immobilization was used postoperatively for 5 days only and the rehabilitation protocol described above was used.

Twelve cases received autologous iliac crest bone graft. There were only slight symptoms at the bone graft donor site in five patients.

The average UCLA score [2] of shoulder function was $18.4 \pm 6.7$ (range 10-28) preoperatively and $29.3 \pm 5.1$ (range 19-35) postoperatively, representing a statistically significant improvement ( $p \leq 0.001)$ (Table 2).

Preoperatively, based on the UCLA score and the Ellman classification [2], two patients had good function, five were rated as satisfactory, and eight as poor. Postoperatively, four patients had excellent function of the shoulder, four had good function, six were rated as satisfactory, and one as poor. This was a case of malunion of a previous proximal humerus fracture.

After 3 months, four patients were asymptomatic with normal function of the operated limb.
The lack of elbow extension averaged $32.5 \pm 20.9^{\circ}$ (range $0-80^{\circ}$ ) preoperatively and $17.1 \pm 15.3^{\circ}$ (range $0-40^{\circ}$ ) postoperatively, representing a statistically significant improvement $(p=0.003)$. Elbow flexion averaged $110.5 \pm 13.9^{\circ}$ (range $80-130^{\circ}$ ) preoperatively and $122.1 \pm 10.9^{\circ}\left(\right.$ range $\left.100-135^{\circ}\right)$ postoperatively, representing a statistically significant improvement $(p=0.001)$ (Table 2).

\section{Discussion}

This case series shows that functional results after humeral shaft nonunion can be obtained with a minimally invasive approach and without bone graft in hypertrophic nonunions. This technique has certain advantages that make it appealing to the trauma surgeon.

Plates can be safely used anteriorly along the humerus with this technique. Good results have been achieved with submuscular plating with no major soft tissue problems and with functional results similar to other methods in the literature. Previous studies demonstrated faster recovery with minimally invasive techniques compared with invasive open technique $[5,7,8]$.

The healing of the humeral shaft fractures in this series presents good results with the advantage of using a minimally invasive technique. This fixation aims at maintaining bone alignment through indirect reduction without an open approach to the fracture site. This preserves the local blood supply and results in less surgical damage to soft tissues, replacing absolute stability by relative stability to achieve bone healing by stimulating bone formation.

There are several studies in the literature showing the advantages of using a bridge-plate in fractures of different parts of the body, such as the femur and the tibia, but there are few reports of use of this technique for humerus [7]. Also, there is a lack of reports in the literature on use of this technique for nonunions.

Being a minimally invasive technique, complications are reduced [7, 8]. There were no nerve injuries. The bridge-plate technique for treatment of humeral shaft nonunion is indicated for cases of both atrophic and hypertrophic nonunion.

A limitation of this study is the inhomogeneous patient population. There is a large age range (23-85 years) with different types of nonunion, and some patients with osteoporotic bones. The objective of the present study is not to compare techniques or healing times between atrophic or hypertrophic nonunions, but rather only to demonstrate that it is possible to use a minimally invasive technique for nonunion and present the results as an alternative to the traditional technique. The lack of a homogeneous population does not influence the results. 

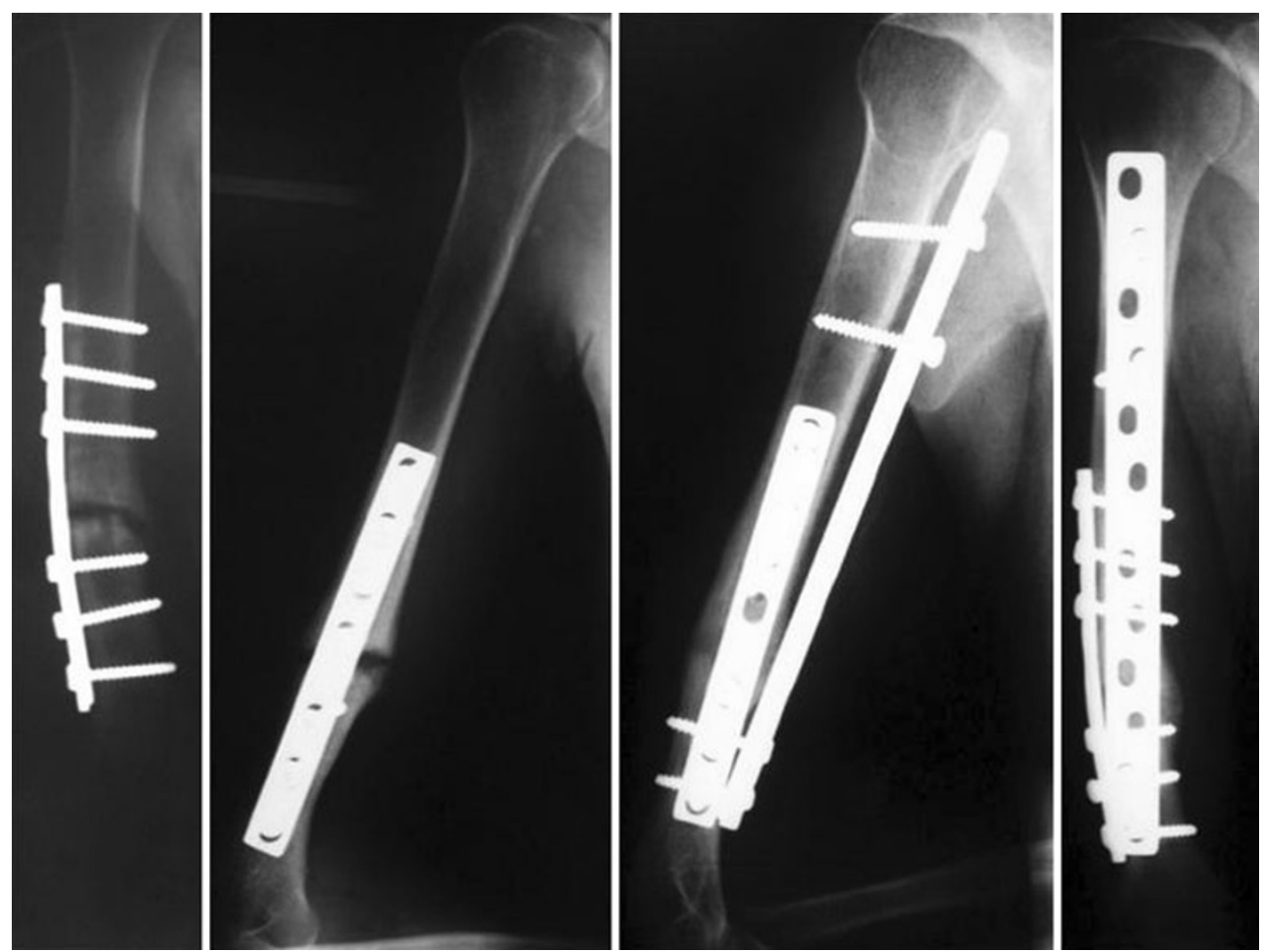

Fig. 4 X-ray images (frontal and lateral views) demonstrate atrophic nonunion instability signs-varus alignment and initial plate bending (left). Six weeks postoperatively with bone formation in the nonunion site (right)
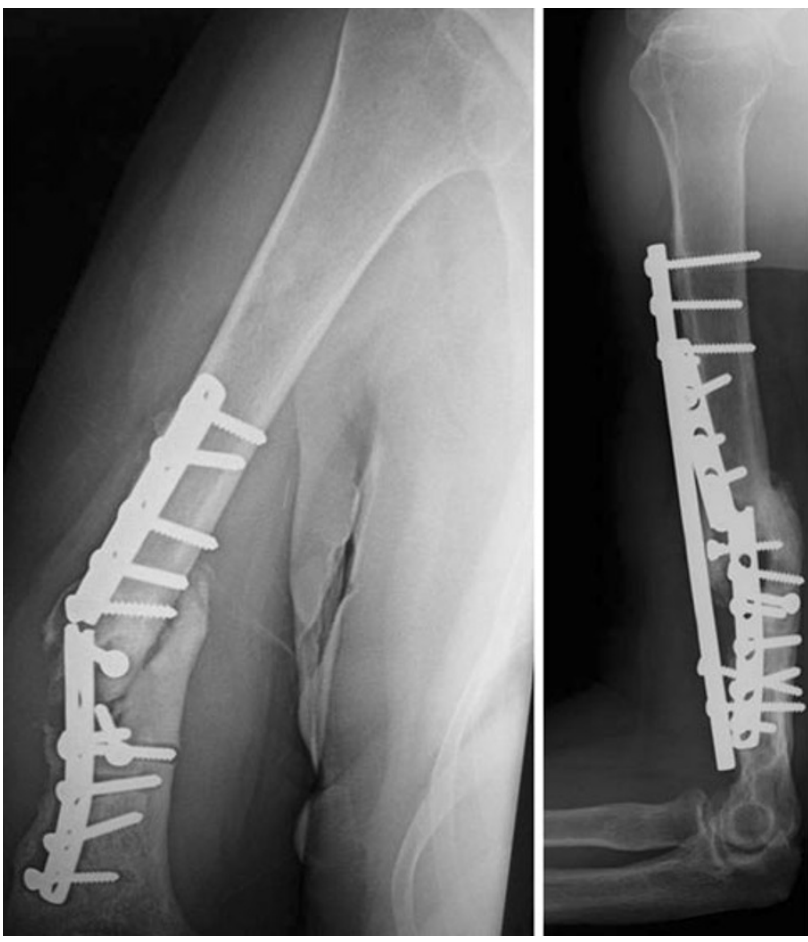

Fig. 5 X-ray images demonstrate atrophic nonunion with previous instability signs $(l e f t)$ healed 6 weeks after the surgery (right)
Use of different plates (DCP or LCP) did not influence the results. Locking screws were used in osteoporotic bones as an alternative for better bone fixation (Fig. 3). The space between distal and proximal fixation was the same as for the DCP fixed with cortical screws. Relative stability was present in both situations. Future studies should compare the two techniques and show their specific indications. Results show that the minimally invasive technique is versatile and can be used with good results for atrophic and hypertrophic nonunions.

It is shown herein that there is a biological capacity for humeral shaft healing achieved through the bridge-plate technique, with use of bone grafting in atrophic cases. It is not necessary to have absolute stability or focal compression, contradicting previous works [5].

Without the need for broad dissection, the local blood supply is preserved. By combining the biological stimulus promoted by the bone graft and the sufficient mechanical stability granted by the plate, all elements necessary for healing of nonunion are present [6].

Following the principle of minimizing invasiveness and according to previous reports $[4,8,9]$, the fixation material was not removed in previously operated patients (Figs. 2, $4,5)$. 
The present technique makes a difficult procedure much easier by avoiding wide dissection, radial nerve isolation, and periosteum stripping.

The results obtained confirm the conclusions of Ring et al. [10], who stated that to achieve bone healing it is important to use a careful, biologically and mechanically adequate technique.

With the plate slid in over the anterior surface of the humerus and respecting the approaches previously described, the radial nerve is totally protected $[1,7]$ and there is no inherent danger to any vascular structure.

It is important, in future studies, to identify the limitations of the technique and define if it is applicable for more severe cases, as well as whether there are possible alternatives to the use of autologous bone graft.

In conclusion, this series demonstrates that the anterior minimally invasive bridge-plate technique for treatment of humeral shaft nonunion presents satisfactory results with regards to bone healing and functional capacity.

\section{Conflict of interest None.}

Open Access This article is distributed under the terms of the Creative Commons Attribution License which permits any use, distribution, and reproduction in any medium, provided the original author(s) and the source are credited.

\section{References}

1. Apivatthakakul T, Patiyasikan S, Luevitoonvechkit S (2010) Danger zone for locking screw placement in minimally invasive plate osteosynthesis (MIPO) of humeral shaft fractures: a cadaveric study. Injury 41:169-172. doi:10.1016/j.injury.2009.08. 002

2. Ellman H, Hanker G, Bayer M (1986) Repair of the rotator cuff. End-result study of factors influencing reconstruction. J Bone Joint Surg Am 68(8):1136-1144

3. Frölke JP, Patka P (2007) Definition and classification of fracture non-unions. Injury 38S:S19-S22. doi:10.1016/S0020-1383(07) 80005-2

4. Gerber A, Marti R, Jupiter J (2003) Surgical management of diaphyseal humeral nonunion after intramedullary nailing: waveplate fixation and autologous bone grafting without nail removal. J Should Elb Surg 12:309-313. doi:10.1016/S1058-2746(03) 00044-2

5. Hierholzer C, Sama D, Toro JB, Peterson M, Helfet DL (2006) Plate fixation of ununited humeral shaft fractures: effect of type of bone graft on healing. J Bone Joint Surg Am 88:1442-1447. doi:10.2106/JBJS.E.00332

6. King AR, Moran SL, Steinmann SP (2007) Humeral nonunion. Hand Clin 23:449-456. doi:10.1016/j.hcl.2007.09.003

7. Livani B, Belangero WD (2004) Bridging plate osteosynthesis of humeral shaft fractures. Injury 35:587-595. doi:10.1016/j.injury. 2003.12.003

8. Marti RK, Verheyen CCPM, Besselaar PP (2002) Humeral shaft nonunion: evaluation of uniform surgical repair in fifty-one patients. J Orthop Trauma 16:108-115. doi:10.1097/00005131-20 0202000-00007

9. Pugh DMW, McKee MD (2003) Advances in the management of the humeral nonunion. J Am Acad Orthop Surg 11:48-59

10. Ring D, Jupiter JB, Quintero J, Sanders RA, Marti RK (2000) Atrophic ununited diaphyseal fractures of the humerus with a bony defect treatment by wave-plate osteosynthesis. J Bone Joint Surg [Br] 82-B:867-871 